\title{
Impact of Nursing Rehabilitation Protocol on Patient`s Satisfaction for Tibial Plateau Fracture Surgery
}

\author{
Mervat Abd El-Fatah Ismael ${ }^{1}$, Zienab Abd-El-lateef Muhammad ${ }^{2}$, Hesham Abd EL- Rahim El-Kady ${ }^{3}$ \& Hala \\ Mohamed Ghanem ${ }^{4}$. \\ 1. Assistant Lecturer, Medical Surgical Nursing Department, Faculty of Nursing, Assiut University, Assiut, Egypt. \\ 2. Professor, Medical Surgical Nursing Department, Faculty of Nursing, Assiut University, Assiut, Egypt. \\ 3. Professor, Orthopedic Surgery Department, Faculty of Medicine, Assiut University, Assiut, Egypt. \\ 4. Assistant Professor, Medical Surgical Nursing Department, Faculty of Nursing, Assiut University, Assiut, Egypt.
} Abstract

Background: A tibial plateau fracture refers to a break in the top of the shin bone, at the knee. Aim: To evaluate the effect of implementing the nursing rehabilitation protocol on Patient's Satisfaction for Tibial Plateau Fracture Surgery. Research design: Quasi-experimental research design. Setting: Trauma unit at Assiut University Hospital. Patient and Methods: Sixty adult patients undergoing tibial plateau fracture surgery. This sample divided into two equal groups (30 patients for each). The study group who received the nursing rehabilitation protocol while the control group received the routine hospital instructions. Tools: I "Patient's health needs assessment sheet ", II "Patient satisfaction with nursing care quality questionnaire ". Results: There was a statistically significant difference (p-value 0,001 ) between the study and control group regarding patient's satisfaction (about $80 \%$ of the study group was satisfied with nursing care quality, $3.3 \%$ of the control group was satisfied with nursing care quality). Conclusion: It was concluded that level of patient's satisfaction for tibial plateau fracture surgery was improved after application of nursing rehabilitation protocol. Recommendations: Simple nursing rehabilitation protocol booklet should be available for patients with tibial plateau fracture surgery to increase the level of patient`s satisfaction.

\section{Keywords: Nursing Rehabilitation Protocol, Satisfaction \& Tibial Plateau Fracture Surgery.}

\section{Introduction}

A tibial plateau fracture is a breakdown or split in the highest point of the tibia. It includes the cartilage surface of the knee joint. This joint helps supports the body weight, and when it is broken, it can't absorb shock (Frank and Sue, 2017).

Treatment plans for tibial plateau fractures rely on its classification. For minor fractures, medical treatment alternatives contain: Resting the knee, bracing the knee, weight-bearing restrictions so as not to harm the joint surface, taking analgesic, applying ice and Resting your limb in an elevated position while the healing process occur (Rachel et al., 2017).

Surgical treatment through open reduction and internal fixation (ORIF), which involves exposing the fracture to direct view after that attaching the bone pieces into place with metal plates and screws. Another technique is external fixation, where wires and pins are put into the bone around the fracture site to safe the fragments in place until they heal. External fixation can be combined with the use of little plates or screws to hold a portion of fracture parts in position; this is called hybrid fixation (McNamara et al., 2015).

Nursing rehabilitation protocol is very imperative to decrease pain, swelling and stiffness, increase muscle strength, and to return the range of movement in the knee. The patient's goals and psychological state must be considered to decrease the risk of persistent pain following surgery (Andrew et al., 2015).

Patient satisfaction is a significant quality result pointer of health care in the hospital setting, and nursing, i.e. patients' satisfaction with nursing service, is an especially significant as a primary determinant of overall satisfaction during a hospital stay (3-5 day). Patient satisfaction with nursing care is the most significant indicator of patients' overall satisfaction with their hospital care (Andaleeb, 2011).

\section{Significance of the study}

While collecting data for my Master's Thesis, it was founded that $33.3 \%$ of patients with lower limb fractures, who developed compartment syndrome, were having fractures of the tibial plateau. After tibial plateau fracture surgery, nursing rehabilitation protocol is needed to increase the level of Patient's Satisfaction and return to the highest level of activity and function.

\section{Aim of the study}

The aim of this study was to evaluate the impact of implementing the nursing rehabilitation protocol on the level of patient's satisfaction for tibial plateau fracture surgery. 


\section{Research Hypothesis}

Patients in the study group will be more satisfied with the quality of care provided than those in the control group.

\section{Subjects \& Methods \\ Research design}

Quasi-experimental research design was utilized in this study.

\section{Setting}

This study was conducted in the trauma unit at Assiut University Hospital.

\section{Sample}

Sixty adult patients (male and female) undergoing tibial plateau fracture surgery who were willing to participate in the study their mean age was $34.47 \pm 9.69$ years old. The sample was calculated by using power analysis according to the patients flow with precision levels $5 \%$ at confidence levels $95 \%$ and $\mathrm{p}<0.05$.

\section{Tools}

Tool I: Patient's health needs assessment sheet:

This tool consisted of three parts and it was used four times (preoperatively, two weeks, one month and six months post operatively).

Part 1: Sociodemographic characteristics of patients: It was developed to assess the patients' socio demographic characteristics. It included age, sex, residence, educational level, occupation, date of admission and date of discharge.

Part (2): Assessment of patient's physical status using the Modified Cincinnati Rating System Questionnaire (Barber SD et al., 2003): It was used to assess patient's physical status this scale includes 8 sections concerning knee assessment. Scoring system of Modified Cincinnati Rating System Questionnaire:- Pain intensity, giving Way and overall activity level ( section 1,3 and 4) scored from (0-20), swelling, walking and stairs (section 2,5 and 6 ) scored from (0-10). Running activity and jumping (section7 and 8) scored from (1-5). Grading the Modified Cincinnati Rating System Questionnaire.

\begin{tabular}{|l|c|}
\hline 1. Poor & $<30$ \\
\hline 2. Fair & $30-54$ \\
\hline 3. Good & $55-79$ \\
\hline 4. Excellent & $>80$ \\
\hline
\end{tabular}

Part (3): Measurement of quadriceps muscle strength: This part was developed for measurement of the patient's quadriceps muscle strength. This was done by using the digital strength measuring device (Beurer Gmbh), while the patient is sitting and a belt is attached for measurement of isometric knee extension strength, a sensor pad is attached to the front of the distal lower extremity by rope $35 \mathrm{~cm}$ in the other side of the machine with a hard object, the patient is asked to push frontally as he can, the power of pushing was translated by number.
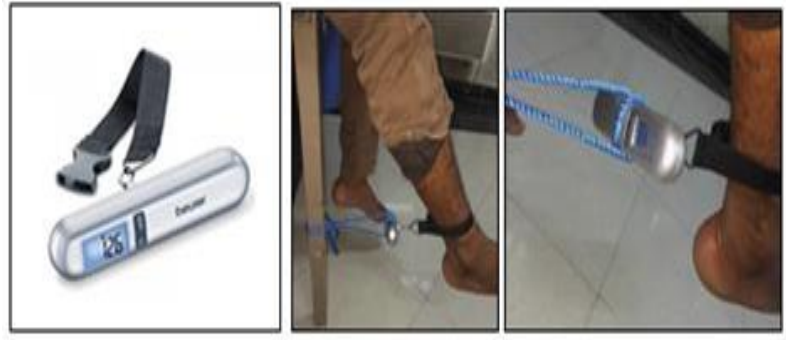

Fig.(1): Beurer Gmbh. Adopted from (https:// www.virtualmarket.ifa-berlin, 2018).

Tool II: Patient satisfaction with nursing care quality questionnaire (PSNCQQ) (Laschinger et al., 2005):

It was designed to measure satisfaction with the overall quality of care during the hospital stay and overall quality of nursing care. The PSNCQQ had 22 questions for entire scale. Besides the basic 19 items, the PSNCQQ contains additional 3 items designed to assess the validity and enable assessment of overall satisfaction with the quality of care and treatment during the hospital stay, overall quality of nursing care and intentions to recommend the hospital to family and friends. The quality of nursing care was graded using a five-point Likert scale, and description intensity ranging from 1 = poor, $2=$ fair, $3=$ good, 4 $=$ very good to $5=$ excellent was used for each item.

\section{Procedure}

This study was carried out in three phases:

\section{I: Preparatory phase}

Tools development

A review of current and past, local and international related literature in the various aspects using books, articles, periodicals and magazines were done.

\section{Content validity and reliability}

Content validity was done by expertise $(2$ medical staff from orthopaedic surgery department) \& (3 nursing staff from the Medical-Surgical Nursing department) who reviewed the tools for clarity, relevance, comprehensiveness, understanding and applicability and then the tools were designed in their final format and tested for reliability using internal consistency for all of the tools which was measured using Cronbach test. The tools proved to be reliable (0. 827 and 0.825 respectively).

\section{A pilot study}

- A pilot study was carried out to test the feasibility, clarity and practicability of the study tools on $10 \%$ of sample (6 cases). It also provided an estimate of time needed to fill out the tools, and those patients were included in the main study as there was no modification. 
- Data were collected from trauma unit at Assiut University Hospital during the period from March 2018 to September 2018.

\section{Ethical consideration}

- Research proposal was approved from ethical committee in the faculty of nursing.

- There is no risk for study subject during application of research.

- An informed consent was obtained from patients to participate in the study and the nature and purpose of the study were explained to them.

- The researcher initially introduced herself to all optional subjects and they were assured that collected data would be absolutely confidential.

- They were informed that participation is voluntary and that they could withdraw at any time of the study.

- Confidentiality of the patient's data was ascertained.

\section{II- Implementation phase}

- The researcher meet with each patient individually and consent for voluntary participation in the study are taken from the patients after the study and its aims were explained to the patient.

- The researcher filled out the patient's health needs assessment sheet. Initial assessment of patient's physical status using the Modified Cincinnati Rating System Questionnaire, measurement of quadriceps muscle strength (Tool I) and measure satisfaction with the overall quality of care during the hospital stay and overall quality of nursing care (Tool II).

- For the study group the researcher was explained to the patient the nursing rehabilitation protocol in one session. The session took about 20-30 minutes. One family member was present in the session for patien't support and increasing their sense of responsibility. Patients were allowed to ask questions in case of misunderstanding while listening and expressing interest.

- After completing the session there were about 510 minutes for discussion and feedback. At the end of the session the researcher emphasized on the importance of follow up visits and arranged with them the time and place for follow up.

\section{III- Evaluation phase}

The last phase of rehabilitation protocol is the evaluation phase. In which the patients was evaluated after two weeks, then one month and six months postoperative for reevaluating the patient's condition for both control and study group by using tool I and tool II.

\section{Statistical analysis}

The data were tested for normality using the Anderson-Darling test and for homogeneity variances prior to further statistical analysis. Categorical variables were described by number and percent $(\mathrm{N}$, $\%)$, where continuous variables described by mean and sandard deviation (Mean, SD). Chi-square test and fisher exact test used to compare between categorical variables where compare between continuous variables by t-test and anova test. A twotailed $\mathrm{p}<0.05$ was considered statistically significant. We are used person correlation to appear the association between scores. All analyses were performed with the IBM SPSS 20.0 software. 


\section{Results}

Table (1): Comparsion between study and control group of the studied patients regarding sociodemographic characteristics ( $\mathbf{n}=\mathbf{3 0}$ for each group).

\begin{tabular}{|c|c|c|c|c|c|}
\hline \multirow{2}{*}{$\begin{array}{l}\text { Sociodemographic } \\
\text { characteristics }\end{array}$} & \multicolumn{2}{|c|}{ Study $(n=30)$} & \multicolumn{2}{|c|}{ Control $(n=30)$} & \multirow{2}{*}{ P. value } \\
\hline & No. & $\%$ & No. & $\%$ & \\
\hline \multicolumn{6}{|l|}{ Age: } \\
\hline $18<30$ years & 13 & 43.3 & 14 & 46.7 & \multirow{4}{*}{0.985} \\
\hline $30<40$ years & 10 & 33.3 & 10 & 33.3 & \\
\hline $40<50$ years & 5 & 16.7 & 4 & 13.3 & \\
\hline $50-60$ years & 2 & 6.7 & 2 & 6.7 & \\
\hline Mean \pm SD & \multicolumn{2}{|c|}{$34.47 \pm 9.69$} & \multicolumn{2}{|c|}{$33.4 \pm 11.29$} & 0.696 \\
\hline \multicolumn{5}{|l|}{ Sex: } & \multirow{3}{*}{0.448} \\
\hline Male & 27 & 90.0 & 25 & 83.3 & \\
\hline Female & 3 & 10.0 & 5 & 16.7 & \\
\hline \multicolumn{5}{|l|}{ Residence: } & \multirow{3}{*}{0.698} \\
\hline Urban & 15 & 50.0 & 14 & 46.7 & \\
\hline Rural & 14 & 46.7 & 16 & 53.3 & \\
\hline \multicolumn{5}{|l|}{ Level of education: } & \multirow{5}{*}{.551} \\
\hline High education & 5 & 16.7 & 5 & 16.7 & \\
\hline Secondary school & 16 & 53.3 & 16 & 53.3 & \\
\hline Read and write & 5 & 16.7 & 2 & 6.7 & \\
\hline Illiterate & 4 & 13.3 & 7 & 23.3 & \\
\hline \multicolumn{5}{|l|}{ Occupation: } & \multirow{3}{*}{0.437} \\
\hline Work & 18 & 60.0 & 14 & 46.7 & \\
\hline Not work & 12 & 40.0 & 16 & 53.3 & \\
\hline
\end{tabular}

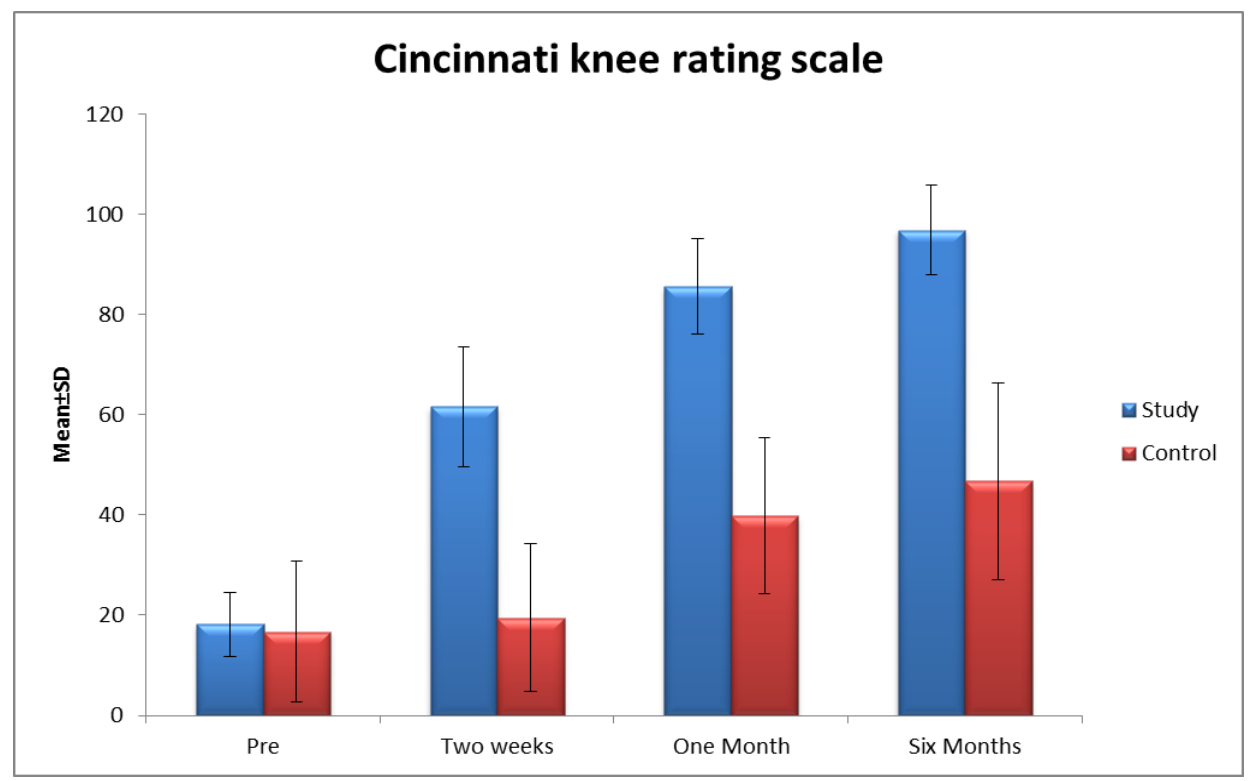

Fig. (1): Percentage distribution of the studied patients (both study and control group) regarding the Cincinnati total mean score $(n=30$ for each group) 
Table (2): Compa rsion between study and control group of the studied patients regarding total mean score of quadriceps muscle strength pre, two weeks, one month and six months post tibial plateau fracture surgery (n $=30$ for each group).

\begin{tabular}{|c|c|c|c|}
\hline $\begin{array}{c}\text { Total mean score of quadriceps } \\
\text { muscle strength }\end{array}$ & $\begin{array}{c}\text { Study } \\
(n=30)\end{array}$ & $\begin{array}{l}\text { Control } \\
(n=30)\end{array}$ & P. value \\
\hline \multicolumn{4}{|l|}{ Affected limb: } \\
\hline Preoperative & $1.52 \pm 0.8$ & $1.18 \pm 0.55$ & 0.066 \\
\hline Two weeks postoperative & $2.15 \pm 0.81$ & $1.2 \pm 0.58$ & $<0.001 * *$ \\
\hline One month postoperative & $4.67 \pm 2$ & $1.65 \pm 0.74$ & $<0.001 * *$ \\
\hline Six months postoperative & $6.35 \pm 2.18$ & $2.1 \pm 1.14$ & $<0.001 * *$ \\
\hline \multicolumn{4}{|l|}{ Un affected limb: } \\
\hline Preoperative & $4.78 \pm 1.85$ & $3.57 \pm 1.1$ & $0.003 * *$ \\
\hline Two weeks postoperative & $5.05 \pm 1.84$ & $3.57 \pm 1.1$ & $<0.001 * *$ \\
\hline One month postoperative & $5.83 \pm 1.85$ & $3.57 \pm 1.1$ & $<0.001 * *$ \\
\hline Six months postoperative & $6.47 \pm 1.96$ & $3.57 \pm 1.1$ & $<0.001 * *$ \\
\hline
\end{tabular}

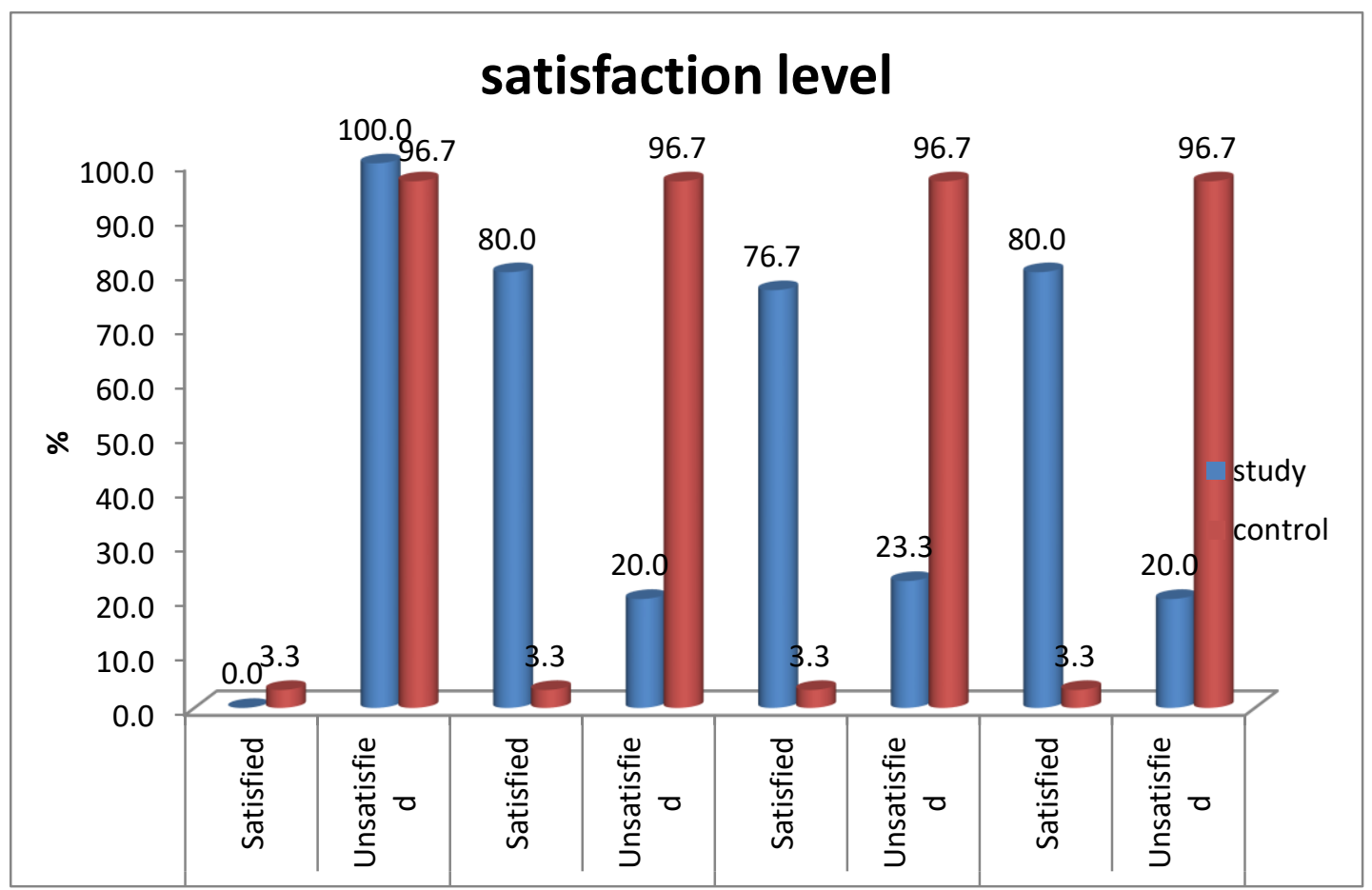

Fig. (2): Percentage distribution of the studied patients (both study and control group) according to level of satisfaction with nursing care quality ( $\mathrm{n}=\mathbf{3 0}$ for each group).

Table (3): Correlation co-efficient between Cincinnati total mean score and level of patient's satisfaction for study and control group during program phases $(n=30$ for each group):

\begin{tabular}{|l|c|c|c|c|}
\hline Correlation & \multicolumn{3}{|c|}{ Level of patient`s satisfaction } \\
\hline \multirow{2}{*}{ Cincinnati total mean score } & \multicolumn{2}{|c|}{ Study } & \multicolumn{2}{c|}{ Control } \\
\cline { 2 - 5 } & $\mathbf{R}$ & $\mathbf{p}$ & $\mathbf{R}$ & $\mathbf{p}$ \\
\hline Preoperative & $-0.777^{* *}$ & $0.001^{* *}$ & $0.634^{* *}$ & $0.001^{* *}$ \\
\hline Two weeks postoperative & 0.297 & 0.111 & $0.586^{* *}$ & $0.001^{* *}$ \\
\hline One month postoperative & 0.015 & 0.937 & $0.635^{* *}$ & $0.001^{* *}$ \\
\hline Six month postoperative & $0.456^{*}$ & $0.011^{*}$ & $0.702^{* *}$ & $0.001^{* *}$ \\
\hline
\end{tabular}


Table (4): Relationship between level of satisfaction of the studied patients and their sociodemographic data during program phases $(n=30$ for each group).

\begin{tabular}{|c|c|c|c|c|c|c|c|c|}
\hline \multirow{3}{*}{$\begin{array}{c}\text { Sociodemographic } \\
\text { data }\end{array}$} & \multicolumn{8}{|c|}{ Level of Satisfaction } \\
\hline & \multicolumn{2}{|l|}{ Pre } & \multicolumn{2}{|c|}{ Two weeks } & \multicolumn{2}{|c|}{ One month } & \multicolumn{2}{|c|}{ Six month } \\
\hline & Mean \pm SD & Range & Mean \pm SD & Range & Mean \pm SD & Range & Mean \pm SD & Range \\
\hline \multicolumn{9}{|l|}{ Age group } \\
\hline 18-30years & $14.15 \pm 1.28$ & $12-15$ & $58.38 \pm 19.99$ & $12-69$ & $59.38 \pm 16.25$ & $12-69$ & $60.77 \pm 15.8$ & $12-69$ \\
\hline $30-40$ years & $13.3 \pm 1.42$ & $12-15$ & $56.5 \pm 22.72$ & $12-69$ & $54.5 \pm 22.66$ & $12-69$ & $56.5 \pm 22.72$ & $12-69$ \\
\hline $40-50$ years & $14.2 \pm 1.3$ & $12-15$ & $46 \pm 29.7$ & $12-69$ & $30.8 \pm 24.8$ & $12-69$ & $35.6 \pm 29.61$ & $12-69$ \\
\hline More than 50 years & $13.5 \pm 2.12$ & $12-15$ & $66.5 \pm 0.71$ & $66-67$ & $66.5 \pm 0.71$ & $66-67$ & $66.5 \pm 0.71$ & $66-67$ \\
\hline P. value & \multicolumn{2}{|c|}{0.457} & \multicolumn{2}{|l|}{0.665} & \multicolumn{2}{|c|}{0.057} & \multicolumn{2}{|c|}{0.136} \\
\hline \multicolumn{9}{|l|}{ Sex } \\
\hline Male & $13.81 \pm 1.36$ & $12-15$ & $55.07 \pm 22.7$ & $12-69$ & $53.93 \pm 21.3$ & $12-69$ & $54.3 \pm 22.64$ & $12-69$ \\
\hline Female & $14 \pm 1.73$ & $12-15$ & $66.67 \pm 0.58$ & $66-67$ & $49.33 \pm 29.74$ & $15-67$ & $66.67 \pm 0.58$ & $66-67$ \\
\hline P. value & \multicolumn{2}{|c|}{0.828} & \multicolumn{2}{|l|}{0.391} & \multicolumn{2}{|c|}{0.734} & \multicolumn{2}{|c|}{0.359} \\
\hline \multicolumn{9}{|l|}{ Residence } \\
\hline Urban & $13.67 \pm 1.45$ & $12-15$ & $46.63 \pm 26.56$ & $12-69$ & $50.13 \pm 21.91$ & $12-69$ & $48.56 \pm 25.19$ & $12-69$ \\
\hline Rural & $13.93 \pm 1.33$ & $12-15$ & $67.21 \pm 1.31$ & $65-69$ & $56.14 \pm 22.33$ & $15-69$ & $63.5 \pm 14.02$ & $15-69$ \\
\hline P. value & \multicolumn{2}{|c|}{0.617} & \multicolumn{2}{|c|}{$0.007 * *$} & \multicolumn{2}{|c|}{0.471} & \multicolumn{2}{|c|}{0.059} \\
\hline \multicolumn{9}{|l|}{ Educational level } \\
\hline High education & $13.6 \pm 1.52$ & $12-15$ & $35.2 \pm 29.09$ & $12-69$ & $47.2 \pm 22.84$ & $12-69$ & $35.2 \pm 29.09$ & $12-69$ \\
\hline Secondary school & $14.25 \pm 1.18$ & $12-15$ & $63.44 \pm 12.99$ & $15-69$ & $62.38 \pm 13.72$ & $15-69$ & $65.38 \pm 5.35$ & $46-69$ \\
\hline Read and write & $13.6 \pm 1.52$ & $12-15$ & $45 \pm 30.16$ & $12-69$ & $34.8 \pm 29.89$ & $12-69$ & $34.6 \pm 29.64$ & $12-69$ \\
\hline Iliterate & $12.75 \pm 1.5$ & $12-15$ & $67.75 \pm 1.5$ & $66-69$ & $49 \pm 24.86$ & $15-69$ & $67.75 \pm 1.5$ & $66-69$ \\
\hline P. value & \multicolumn{2}{|c|}{0.236} & \multicolumn{2}{|c|}{$0.022 *$} & \multicolumn{2}{|c|}{0.063} & \multicolumn{2}{|c|}{$0.001 * *$} \\
\hline \multicolumn{9}{|l|}{ Occupation } \\
\hline Working & $13.67 \pm 1.41$ & $12-15$ & $57.94 \pm 20.28$ & $12-69$ & $58.56 \pm 17.96$ & $12-69$ & $57.94 \pm 20.28$ & $12-69$ \\
\hline Not working & $14.08 \pm 1.31$ & $12-15$ & $53.67 \pm 24.57$ & $12-69$ & $45.83 \pm 25.18$ & $12-69$ & $51.92 \pm 24.28$ & $12-69$ \\
\hline P. value & \multicolumn{2}{|c|}{0.423} & \multicolumn{2}{|l|}{0.607} & \multicolumn{2}{|l|}{0.117} & \multicolumn{2}{|c|}{0.467} \\
\hline
\end{tabular}

Table (1): Shows that the majority of the studied patients were males $(83.3 \%)$, their age ranged from 18 to $<30$ years and living in rural areas in both study and control group, as regard level of education; the highest percentages in both study and control group were secondary educated $(53.3 \%)$ and workers $(60.0 \%, 46.7)$ respectively.

Figure (1): Shows that there was a high statistically significant difference between the study and the control group regarding total score of the modified Cincinnati knee rating scale two weeks, one month and six months post tibial plateau fracture surgery.

Table (2): Illustrates that there was a high statistically significant difference between the study and the control group regarding total mean score of quadriceps muscle strength two weeks, one month and six months post tibial plateau fracture surgery.

Figure (2): Shows that there was a statistical significant difference between the study and control group regarding satisfaction level after implementation of nursing rehabilitation protocol two weeks, one month and six months tibial plateau fracture surgery.
Table (3): Illustrates that there was a positive correlation with significant statistical differences ( $\mathrm{p}<$ 0.01 ) between Cincinnati total mean score and level of satisfaction after implementation of nursing rehabilitation protocol for study and control group during program phases.

Table (4): Illustrates that no relation between age, sex, occupation and level of satisfaction after implementation of nursing rehabilitation protocol and there is relation between residence, educational level and level of satisfaction.

\section{Discussion}

Regarding socio-demographic characteristics of the patients; the result of the present study revealed that, most of the studied patients their age ranged from more than eighteen to less than thirty years. This result disagree with (Biju et al., 2014) who carried out A prospective study was conducted at Department of Orthopedics, Narayana Medical College Hospital, Nellore, Andhra Pradesh, India for the period of 20 months from August 2010 to April 
2012 which revealed that the mean age of the studied patients was 41 years.

The current study revealed that, the majority of the studied patients were males. This study finding was in line with (Suélem et al., 2008) which conducted at surgical department, between 2002 and 2005 at the Hospital das Clínicas, Campinas State University (HC - UNICAMP). Entitled as assesment of the functional capacity of individuals submitted to surgical treatment after tibial plateau fracture which revealed that; the majority of the study group were males.

In the current study; it was founded that the majority of the studied patients were secondary educated, workers and live in rural area. which was in line with (Berkson \& Virkus, 2006) which conducted at Department of Orthopaedic Surgery, Rush University Medical Center, Chicago, USA. Entitled as Highenergy tibial plateau fractures which revealed that; the majority of the studied patients stayed in hospital for 7 days.

Regarding assessment of patient's physical status; the results of the present study illustrated that, there was a statistically significant difference between the study and control group regarding total mean scores of the Cincinnati knee rating scale collectively in the follow up periods. This result was supported by (Michael et al., 2015) who carried out a study in Toronto - Canada entitled as Distal femoral varus osteotomy combined with tibial plateau fresh osteochondral allograft for post-traumatic osteoarthritis of the knee which revealed that a significant difference in the knee function scores.

Regarding measurement of quadriceps muscle strength; the results of the present study illustrated that, there was a statistically significant difference between the study and control group regarding measurement of quadriceps muscle strength in the follow up periods. This study finding was supported by (Gaston et al., 2005) who carried out a prospective study for patients admitted to trauma center Between May 1996 and December 2001,who were diagnosed with an isolated fracture of the tibial plateau entitled as Recovery of knee function following fracture of the tibial plateau which revealed that a significant difference in measurement of quadriceps muscle strength.

Regarding the level of patient's satisfaction; the results of the present study illustrated that, there was a statistically significant difference (p-value 0,001) between the study and control group regarding satisfaction level after implementation of nursing rehabilitation protocol in the follow up periods. This result explained the effect of implementation of nursing rehabilitation protocol and its importance in improving patient's condition and their level of satisfaction with the overall quality of nursing care.

Regarding relations (Correlation co-efficient between Cincinnati total mean score and level of satisfaction for study and control group during program phases) ; the present study revealed that there was a positive correlation with significant statistical differences between Modified Cincinnati Rating System scale and satisfaction level after implementation of nursing rehabilitation protocol for study and control group two weeks, one month and six months tibial plateau fracture surgery

The present study revealed that no relation between age, sex, occupation and level of patient's satisfaction after implementation of nursing rehabilitation protocol and there is relation between residence, educational level and level of satisfaction. This may be due to that the educational level increase in people's alertness about importance of being knowledgeable and what should be done to maintain or enhance their health.

\section{Conclusion}

Based on the results of the present study, it can be concluded that; providing written and illustrated nursing rehabilitation protocol for patients was much more effective on patient's satisfaction after tibial plateau fracture surgery than those patients in the control group who received resident's oral instructions.

\section{Recommendation}

The study recommended that simple nursing rehabilitation protocol booklet should be available for patients with tibial plateau fracture surgery to increase the level of patient's satisfaction.

\section{References}

1. Andaleeb S., (2011): Service quality and patient satisfaction, A study of Hospitals in a developing country." Social Science Medicine, V: (52), N: (70), P.P: (1359).

2. Andrew D., Paolucci E., \& Korley R., (2015): The effect of three-dimensional computed tomography reconstructions on preoperative planning of tibial plateau fractures: a casecontrol series. BMC Musculoskeletal Disorders, V: (16), N: (10), P.P: (144).

3. Berkson E., \& Virkus W., (2006): Highenergy tibial plateau fractures. Journal of American Academy of Orthopedic Surgeons, V (14), N (1), P.P (20-31). 
4. Beurer Gmbh (2018): Adopted from https:// www.virtualmarket.ifa-berlin. Accessed at 12/6/2018 at 6 p.m.

5. Biju R., Babu K., Rallapalli R., \& Shaik M., (2014): An outcome of surgical management of the tibial plateau fractures, $\mathrm{V}$ (3), N (2), P.P (110-114).

6. Frank R., \& Sue D., (2017): In Noyes' Knee Disorders: Surgery, Rehabilitation, Clinical Outcomes, $2^{\text {nd }}$ ed, Ch (3), P.P (344-372).

7. Gaston, E., \& Keating J., (2005): Recovery of knee functions following fracture of the tibial plateau. The Bone \& Joint Journal, V (87), N (9), P.P (27).

8. McNamara I., Smith T., Shepherd K., Clark A., Nielsen D., Donell S., \& Hing C., (2015): Surgical fixation methods for tibial plateau fractures. Cochrane Database of Systematic Review. V (9), N (3), P.P (1009).

9. Michael D., Gross A., Dwyer T., Safir O., Backstein D., Chaudhry H., Goulding A., \& Kosashvili Y., (2015): Distal femoral varus osteotomy combined with tibial plateau fresh osteochondral allograft for post-traumatic osteoarthritis of the knee Surgery. Sports Traumatology, Arthroscopy Journal, V (23), N (5), P.P (1317-1323).

10. Rachel M., Forsythe B., \& Matthew T., (2017): Case Competencies in Orthopaedic Surgery Book, Ch: 28, P.P: (277).

11. Suélem P., Lopes R., Carvalho M., Ferreira A., Rodrigo C., \& Regazzo P., (2008): Assesment of the functional capacity of individuals submitted to surgical treatment after tibial plateau fracture. Acta Ortopédica Brasileira Journal, V (16), N (3), P.P (168-172).

12. Barber S., Noyes F., \& Mooar L., (2003): A prospective, randomised comparison of autologous chondrocyte implantation versus mosaicplasty for osteochondral defects in the knee. Bone Joint Surgery Journal. V (85), N (2), P.P (30-223). 